\title{
1 An international report on bacterial communities in esophageal squamous cell
}

carcinoma

4 Jason Nomburg ${ }^{1,2,3}$, Susan Bullman ${ }^{4}$, Dariush Nasrollahzadeh ${ }^{5,6}$, Eric A. Collisson ${ }^{7,8}$,

5 Behnoush Abedi-Ardekani ${ }^{6}$, Larry O. Akoko ${ }^{9}$, Joshua R. Atkins ${ }^{6}$, Geoffrey C. Buckle ${ }^{7,8}$,

6 Satish Gopal ${ }^{10}, \mathrm{Nan} \mathrm{Hu}^{11}$, Bongani Kaimila ${ }^{12}$, Masoud Khoshnia ${ }^{5}$, Reza Malekzadeh ${ }^{5}$,

7 Diana Menya ${ }^{13}$, Blandina T. Mmbaga ${ }^{14,15}$, Sarah Moody ${ }^{16}$, Gift Mulima ${ }^{17}$, Beatrice P.

8 Mushi $^{9}$, Julius Mwaiselage ${ }^{18}$, Ally Mwanga ${ }^{9}$, Yulia Newton ${ }^{19}$, Dianna L. $\mathrm{Ng}^{7,20}$, Amie

9 Radenbaugh ${ }^{19}$, Deogratias S. Rwakatema ${ }^{14,15}$, Msiba Selekwa ${ }^{9}$, Joachim Schüz ${ }^{21}$,

10 Philip R. Taylor ${ }^{11}$, Charles Vaske ${ }^{19}$, Alisa Goldstein ${ }^{11}$, Michael R. Stratton ${ }^{16}$, Valerie

11 McCormack ${ }^{21}$, Paul Brennan ${ }^{6}$, James A. DeCaprio ${ }^{1,3,22}$, Matthew Meyerson ${ }^{1,2,22,23^{*}}$, Elia

12 J. Mmbaga9 ${ }^{9}, 4^{*}$, Katherine Van $\operatorname{Loon}^{7,8^{*}}$

151 Department of Medical Oncology, Dana-Farber Cancer Institute, Boston, MA

162 Broad Institute of MIT and Harvard, Cambridge, MA

173 Harvard Program in Virology, Harvard Medical School, Boston, MA

184 Fred Hutchinson Cancer Research Center, Seattle, Washington, USA

195 Digestive Oncology Research Center, Digestive Disease Research Institute, Tehran

20 University of Medical Sciences, Shariati Hospital. Tehran Iran.

216 International Agency for Research on Cancer (IARC/WHO), Genomic Epidemiology

22 Branch, Lyon, France 
237 University of California, San Francisco (UCSF) Helen Diller Family Comprehensive

24 Cancer Center, San Francisco, CA, USA

258 Division of Hematology/Oncology, Department of Medicine, UCSF, San Francisco,

26 California, USA

279 Muhimbili University of Health and Allied Sciences, Dar es Salaam, Tanzania

2810 University of North Carolina, Chapel Hill, North Carolina, USA

2911 Division of Cancer Epidemiology and Genetics, National Cancer Institute, Bethesda,

30 MD, USA

3112 UNC Project - Lilongwe, Malawi

3213 School of Public Health, Moi University, Eldoret, Kenya

3314 Kilimanjaro Clinical Research Institute, Kilimanjaro Christian Medical Centre, Moshi,

34 Tanzania

3515 Kilimanjaro Christian Medical University College, Moshi, Tanzania

3616 Cancer, Ageing and Somatic Mutation, Wellcome Trust Sanger Institute, Wellcome

37 Trust Genome Campus, Hinxton, Cambridgeshire, UK

3817 Kamuzu Central Hospital, Lilongwe, Malawi

3918 Ocean Road Cancer Institute, Dar es Salaam, Tanzania

4019 NantOmics/NantHealth, Inc., El Segundo, California, USA

4120 Department of Pathology, UCSF, San Francisco, CA, USA

4221 International Agency for Research on Cancer (IARC/WHO), Environment and Lifestyle

43 Epidemiology Branch, Lyon, France

4422 Department of Medicine, Brigham and Women's Hospital, Harvard Medical School, 45 Boston, MA 
4623 Department of Genetics, Harvard Medical School, Boston, MA

4724 Department of Community Medicine and Global Health, University of Oslo, Norway

50 *Correspondence to:

51 Katherine Van Loon - Katherine.VanLoon@ucsf.edu

52 Elia J. Mmbaga - eliajelia@yahoo.co.uk

53 Matthew Meyerson - matthew_meyerson@dfci.harvard.edu

54

55 


\section{ABSTRACT}

57 The incidence of esophageal squamous cell carcinoma (ESCC) is disproportionately

58 high in the eastern corridor of Africa and parts of Asia. Emerging research has identified

59 a potential association between poor oral health and ESCC. One proposed biological

60 pathway linking poor oral health and ESCC involves the alteration of the microbiome.

61 Thus, we performed an integrated analysis of four independent sequencing efforts of

62 ESCC tumors from patients from high- and low-incidence regions of the world. Using

63 whole genome sequencing (WGS) and RNA sequencing (RNAseq) of ESCC tumors

64 and WGS of synchronous collections of saliva specimens from 61 patients in Tanzania,

65 we identified a community of bacteria, including members of the genera Fusobacterium,

66 Selenomonas, Prevotella, Streptococcus, Porphyromonas, Veillonella, and

67 Campylobacter, present at high abundance in ESCC tumors. We then characterized the

68 microbiome of $238 \mathrm{ESCC}$ tumor specimens collected in two additional independent

69 sequencing efforts consisting of patients from other high-ESCC incidence regions

70 (Tanzania, Malawi, Kenya, Iran, China). This analysis revealed a similar tumor

71 enrichment of the ESCC-associated bacterial community in these cancers. Because

72 these genera are traditionally considered members of the oral microbiota, we explored if

73 there is a relationship between the synchronous saliva and tumor microbiomes of ESCC

74 patients in Tanzania. Comparative analyses revealed that paired saliva and tumor

75 microbiomes are significantly similar with a specific enrichment of Fusobacterium and

76 Prevotella in the tumor microbiome. Together, these data indicate that cancer-

77 associated oral bacteria are associated with ESCC tumors at the time of diagnosis and

78 support a model in which oral bacteria are present in high abundance in both saliva and 
bioRxiv preprint doi: https://doi.org/10.1101/2021.09.29.462325; this version posted September 29, 2021. The copyright holder for this preprint (which was not certified by peer review) is the author/funder, who has granted bioRxiv a license to display the preprint in perpetuity. It is made available under aCC-BY 4.0 International license.

79 tumors of ESCC patients. Longitudinal studies of the pre-diagnostic oral microbiome are

80 needed to investigate whether these cross-sectional similarities reflect temporal

81 associations.

82

83 


\section{INTRODUCTION}

85 Esophageal cancer is the sixth most common cause of cancer-related death worldwide

86 (1).There are two histologic subtypes of esophageal cancer with distinct biological

87 characteristics, geographic distributions, and risk factors (2). Esophageal

88 adenocarcinoma is the most common histologic form of esophageal cancer in high-

89 income countries and is associated with factors including gastroesophageal reflux

90 disease, Barrett's esophagus, and obesity $(3,4)$. By contrast, esophageal squamous

91 cell carcinoma (ESCC) represents more than $90 \%$ of worldwide esophageal cancer

92 cases and is the dominant histology in low-resource settings. In particular, there are two

93 main regions where ESCC is endemic: (1) the Asian esophageal cancer belt, extending

94 from western/northern China to central and southeast Asia; and (2) the eastern corridor

95 of Africa, extending from Ethiopia to South Africa $(5,6)$.

97 Emerging research has identified a possible association between poor oral health and

98 ESCC. Studies from Asia, Europe, Latin America, Kenya, and Iran have reported

99 associations of ESCC with poor oral hygiene, chronic periodontal disease, dental decay,

100 and tooth loss (7-16). Recently, three parallel case-control studies in Kenya and

101 Tanzania, conducted as part of the African Esophageal Cancer Consortium (AfrECC)

102 and ESCCAPE (esccape.iarc.fr) collaborations, reported possible associations of poor

103 or infrequent oral hygiene with increased risk for ESCC in East Africa (17-20).

104

105 Alterations of the oral microbiome due to poor oral health is one proposed biological

106 pathway that could explain the link between oral health and ESCC. Many bacterial 
107 genera associated with gastrointestinal cancers contain species that are traditionally

108 associated with healthy or diseased oral microbiomes. For example, Helicobacter pylori

109 was discovered to be associated with gastric cancers and mucosa-associated lymphoid

110 tissue (MALT) lymphomas, indirectly by promoting gastric inflammation and directly by

111 influencing cellular signaling (21). Similarly, bacteria of the genera Fusobacterium,

112 Selenomonas, and Prevotella are enriched in colorectal cancers (22-24) and can be

113 visualized invasively within tumor tissue (25). Fusobacterium, in particular, has been

114 reported to promote carcinogenesis through the selective expansion or inhibition of

115 certain classes of immune cells (26) and may drive cellular proliferation by stimulating

116 Wnt/ $\beta$-catenin signaling $(27,28)$. Other bacterial genera such as Porphyromonas,

117 Campylobacter, and Streptococcus have emerging associations with various human

118 gastrointestinal cancers (29-35).

120 As part of ongoing investigation into the microbiome's association with ESCC, we

121 performed an integrated analysis of four independent sequencing efforts including

122 ESCC tumors from patients from both high- and low-incidence regions of the world. In

123 addition, we investigated the relationship between the microbiomes of matched ESCC

124 tumors and saliva specimens in a subset of ESCC cases.

\section{RESULTS}

\section{Study Population}

128 To evaluate the potential role of the host microbiota in ESCC, we investigated the 129 microbiome of 299 ESCC specimens from patients in five different countries with a high 
130 incidence of ESCC. Specimens were collected through four independent sequencing

131 efforts (Figure 1A). Specimens consisted of whole genome sequencing (WGS) and

132 RNA sequencing (RNAseq) data from the tumor and saliva of 61 patients from Tanzania

133 (the "MUHAS Tanzania" cohort) (36), RNAseq data from the tumors of 30 ESCC

134 patients in Malawi (the "UNC Project - Malawi” cohort) (37), and WGS from 208

135 additional samples of tumors from patients in high ESCC incidence regions, including

136 specimens from ESCC patients in Tanzania $(n=18)$ and Kenya $(n=64)$ that were

137 collected in the ESCCAPE studies (esccape.iarc.fr) and specimens from ESCC patients

138 in East Golestan, Iran $(n=55)$ and Shanxi, China $(n=71)$ that were sequenced as part of

139 the Cancer Research UK Mutographs project ("Mutographs" cohorts) (38). In addition,

140 we analyzed WGS data of ESCC from The Cancer Genome Atlas (39), which includes a

141 small number of tumors from patients in low-incidence geographic regions including the

142 United States $(n=3)$, Ukraine $(n=3)$, Vietnam $(n=22)$, and Russia $(n=8)$ (the "TCGA"

143 cohort). Patient characteristics are shown in Table 1.

145 Bacterial populations are abundant and diverse in ESCC tumors

146 We used the metagenomic analysis tool GATK-PathSeq (40) to process the RNAseq

147 and WGS data. GATK-PathSeq uses a sequential mapping strategy to assign reads to

148 human and microbial reference genomes, resulting in detailed information on

149 sequencing reads of human and microbial origin (Figure S1A). We likewise used

150 GATK-PathSeq to process WGS data sets from 50 colon adenocarcinoma (COAD)

151 specimens available from TCGA (41) for comparison, as there is strong evidence of

152 microbial associations with COAD (22-25). 
154 The bacterial burden of ESCC tumors ranged from 10 to 1000 bacterial reads per

155 million human reads, similar to numbers observed in TCGA COAD (Figure 1B).

156 Furthermore, the Shannon diversity of bacterial populations at the genus level ranged

157 from 2 to 3 (Figure 1C). By comparison, ESCC-associated bacterial communities are as

158 diverse or more diverse than TCGA COAD. At the phylum level, ESCC bacterial

159 populations generally consist of Firmicutes, Bacteroidetes, Proteobacteria,

160 Actinobacteria, and Fusobacteria (Figure 1D, Figure S1B). Of note, the higher than

161 expected abundance of the phylum Actinobacteria specifically in the TCGA ESCC

162 samples is attributable, in particular, to a very high abundance of the genus

163 Tetrasphaera (Figure S1C). This is evidenced by a depressed Shannon diversity of

164 Actinobacteria genera in these samples (Figure S1D) and may indicate contamination

165 of the TCGA ESCC samples. Actinobacteria have been reported as a source of

166 contaminating reads in TCGA gastrointestinal cancer samples (42).

168 Bacterial genera associated with carcinogenesis are observed at high relative

\section{9 abundance in ESCC tumors from Tanzania}

170 To determine if bacteria with known associations with cancer are present in ESCC, we

171 first analyzed the sequencing series of the 61 ESCC cases from the MUHAS Tanzania

172 cohort with both WGS and RNAseq data. The paired WGS and RNAseq data from

173 these tumors allowed investigation of bacterial communities at the DNA and RNA levels.

174 Both WGS and RNAseq data revealed high relative abundance of bacterial genera

175 previously associated with carcinogenesis in these ESCC tumors (Figure 2A, 2B). The 
176 high relative abundance of the Fusobacterium genus was particularly notable. Other

177 bacterial genera of interest include Streptococcus, Porphyromonas, Campylobacter,

178 Prevotella, Veillonella, and Selenomonas, many of which have been associated with

179 gastrointestinal malignancies alongside or independently of Fusobacterium (25, 29, 32,

$18034,43)$. The mean Jaccard similarity index between tumor RNAseq and WGS data from

181 the same tumor is 0.54 , greater than the average Jaccard similarity index of random

182 RNAseq-WGS pairs (0.36), indicating that bacterial populations inferred from WGS and

183 RNAseq data are generally consistent (Figure 2C).

185 Next, we attempted to determine if similar bacterial genera were also present in ESCC

186 from patients in high-incidence countries beyond Tanzania. Investigation of RNA

187 sequencing data from patients in Malawi, WGS data from patients in Kenya, China, and

188 Iran, as well as from the independent ESCCAPE Tanzania patient group revealed

189 pervasive evidence of similar bacterial genera in the tumors of these patients (Figure

190 2D, Figure S2A). To investigate if similar microorganisms were found in ESCC tumors

191 from patients in low-incidence regions, we investigated WGS data from ESCC tumors

192 originating from USA, Ukraine, Vietnam, and Russia that were available through TCGA.

193 While the number of samples available from low-incidence regions is low and relies on a

194 single sequencing effort, we found that the tumors of many of these patients contain

195 similar bacterial genera (Figure 2D, Figure S2A). Colon cancers from the TCGA COAD

196 cohort revealed evidence of Fusobacterium, as expected; however, these COAD

197 samples were notable for much lower relative abundance of the other genera of interest,

198 when compared to ESCC tumors. 
200 Evaluation of association between saliva and tumor microbiomes in ESCC

201 patients from Tanzania

202 We next investigated the similarity between the saliva and tumor microbiomes of ESCC

203 patients. Paired saliva samples were only available from patients in the MUHAS

204 Tanzania cohort $(\mathrm{N}=45)$; these paired saliva specimens were analyzed to evaluate

205 bacterial abundance as a proxy for the oral microbiome.

207 We first assessed the similarity between paired saliva and tumor microbiomes with the

208 Bray Curtis similarity index (44). To avoid potential confounding due to low bacterial

209 read counts in some tumor samples, we limited these analyses to the 21 tumor-saliva

210 pairs that contain appreciable microbial sequencing depth (at least 10,000 bacterial

211 reads each). We found that the saliva and tumor microbiomes from the same patient in

212 the Tanzanian samples are significantly more similar than random saliva-tumor pairs

$213(p=0.0003$, Wilcoxon rank sum test) (Figure 3A). Next, we asked if there are bacterial

214 genera whose relative abundance in the saliva correlates with their relative abundance

215 in the tumor. For this analysis, we included only common-abundant bacterial genera

216 with at least $1 \%$ relative abundance in at least three tumor-oral pairs. The relative

217 abundance of four bacterial genera (Fusobacterium, Veillonella, Streptococcus, and

218 Porphyromonas) are strongly correlated between tumor and saliva microbiomes, while

219 other common-abundant bacterial genera were not (Figure 3B). To assess if any

220 bacterial genera are preferentially enriched in the tumor microbiome relative to the

221 saliva microbiome, we next calculated the difference in the relative abundance of the 
222 common-abundant bacterial genera between saliva-tumor pairs. Several genera

223 including Porphyromonas and Veillonella were at higher relative abundance in the

224 saliva, while Prevotella and Fusobacterium were enriched in the tumor microbiome

225 (Figure 3C). Finally, the relative abundance of tumor-associated bacteria including

226 Fusobacterium, Prevotella, Selenomonas, Veillonella, Streptococcus, and

227 Campylobacter are strikingly similar between the microbiomes of tumor and oral pairs

228 (Figure 3D). Altogether, these data support the hypothesis that there is an association

229 between the oral and tumor microbiome of ESCC patients in Tanzania.

\section{DISCUSSION}

232 This report provides an analysis of bacterial communities present in ESCC tumors from

233 nine countries from different regions of the world, analyzed in four independent

234 sequencing efforts. We found traditionally oral, cancer-associated, bacterial genera in

235 tumors from patients in Tanzania, Malawi, Kenya, China, and Iran. These results

236 provide evidence that these bacterial genera may be associated with ESCC in high-

237 incidence regions. We also identified similar bacterial genera in ESCC tumors from low-

238 incidence regions, although this finding is based on a small sample size and only one

239 sequencing cohort. Finally, in a sub-analysis of tumor and saliva pairs available from

240 Tanzania, we demonstrated that the synchronous collected saliva and tumor

241 microbiomes of ESCC patients are strikingly similar at the time of diagnosis; in

242 particular, we identified a specific correlation between the saliva and tumor relative

243 abundance of the bacterial genera Fusobacterium, Veillonella, Streptococcus, and 
244 Porphyromonas, with Prevotella and Fusobacterium significantly enriched in the tumor

245 microbiome.

247 Many of the bacterial genera identified in this study have been previously implicated in

248 the carcinogenesis of gastrointestinal cancers. For example, studies have found that

249 oral microbiota including Fusobacterium, Prevotella, Selenomonas, Veillonella,

250 Streptococcus, and Campylobacter can be used to distinguish individuals with colorectal

251 cancer from healthy controls (45), and that Fusobacterium nucleatum strains that

252 colonize the oral cavity and tumors of patients with colorectal cancer are identical in

253 some patients (46), raising the possibility that the oral cavity is a source of extra-oral

254 cancer microbiota. Our group has previously shown that Fusobacterium, Selenomonas,

255 and Prevotella can be visualized invasively within colorectal tumors and liver

256 metastases (25). Fusobactium nucleatum has been previously identified in esophageal

257 cancers and is associated with shorter survival (47). Members of the genus

258 Porphyromonas have been previously observed invasively within ESCC tumors (29)

259 and have been reported to promote oral squamous cell carcinoma through a variety of

260 mechanisms $(30,31)$. Campylobacter jejuni has been reported to promote

261 tumorigenesis in mice (32), and Streptococcus species have been identified in human

262 esophageal cancers (33). In addition, the striking association of Streptococcus bovis

263 with colorectal cancer has led to the recommendation that colonoscopy be performed

264 upon detection of Streptococcus bovis bacteremia or endocarditis $(34,35)$. Oral

265 commensal bacteria such as Veillonella species have been previously implicated in

266 pathogenesis of lung cancer (43). A prospective cohort of American patients (48) and a 
267 study of Japanese patients (49) likewise found that oral microbiome composition reflects

268 risk of esophageal cancers

270 We found that bacterial genera including Fusobacterium, Prevotella, Selenomonas,

271 Veillonella, Streptococcus, and Campylobacter are pervasive in the microbiome of

272 ESCC tumors from patients in high-incidence regions. Moreover, the bacterial

273 composition of ESCC tumors is remarkably similar across countries in those high-

274 incidence regions, raising the possibility that these bacterial genera may be involved in

275 ESCC carcinogenesis or that they may colonize tumors as a result of the common

276 clinical presentation of patients with severe dysphagia. Notably, there are several

277 alternative hypotheses that warrant mention. For example, it is possible that the ESCC-

278 associated bacterial genera simply represent common members of the esophageal

279 microbiome (50) and that the microbial populations we observed in these cancers are

280 not significantly different from those found in normal esophagus tissue. A limitation of

281 our study is a lack of normal esophageal tissue from ESCC cases or healthy controls in

282 these settings, which would allow us to address this possibility. Another possible

283 explanation is that ESCC tumors provide a favorable niche in which these bacteria are

284 sequestered and allowed to colonize due to the propensity of this disease to cause

285 malignant obstruction. Thus, it is plausible that ESCC-associated bacteria are not

286 necessarily promoting ESCC carcinogenesis but rather represent passengers resulting

287 from the sequestration of oral secretions proximal to an obstructing tumor. While the

288 previous association of these bacterial genera with other cancers is consistent with the

289 hypothesis that they influence carcinogenesis of ESCC, future studies are necessary to 
290 identify which, if any, direct influences these bacterial genera have upon ESCC

291 carcinogenesis. Nevertheless, even if these bacterial genera do not have a role in

292 increasing ESCC risk, but arise at the time of disease onset, they may have an

293 important role to play as part of a non-invasive early-detection biomarker. Finally, a

294 concern of all microbiome analyses is that observed bacteria can be a consequence of

295 contamination at some step between tumor harvest and sequencing. While some TCGA

296 samples may be contaminated by Actinobacteria as previously noted, the presence of

297 Fusobacterium, Prevotella, Selenomonas, Veillonella, Streptococcus, and

298 Campylobacter in four independently collected cohorts indicates that these finding are

299 unlikely due to contamination.

301 While this study focused on the presence of bacteria with ESCC in high-incidence

302 regions, we found evidence of similar cancer-associated bacteria in tumors in patients

303 from low-incidence regions (USA, Ukraine, Vietnam, and Russia). A limitation of this

304 assessment is the small sample size $(n=36)$ and reliance on a single TCGA cohort that

305 likely contains contaminants (42). Regardless, this finding does not exclude the

306 possibility that the microbiome could be a factor driving patterns of ESCC incidence. For

307 example, it is possible that the prevalence of ESCC-associated bacteria in people could

308 vary across regions, which in turn could drive these differing rates of ESCC incidence.

309 This is an important topic for future study.

310

311 We found that the structure of synchronous paired tumor and oral microbiomes were

312 strikingly similar. It is possible that this similarity is driven by transient contact of saliva 
313 and its associated microbiome with the tumor (e.g., during swallowing or tumor

314 extraction). However, we found that only four of sixteen common-abundant bacterial

315 genera correlate in abundance between the tumor and oral microbiomes, suggesting

316 tumor-oral microbiome similarity is not driven exclusively by "in-trans" interactions

317 between the saliva and tumor. We also found that genera including Prevotella and

318 Fusobacterium are often specifically enriched in the tumor microbiome, supporting a

319 model where specific oral bacterial preferentially colonize the tumor. A caveat of this

320 study is that we infer oral bacterial populations from the saliva, despite diverse

321 communities of bacteria throughout the oral cavity (51). However, we do observe

322 Fusobacterium in the saliva despite its general association with periodontal plaques

323 (52), suggesting saliva is capable of detecting periodontal pathogens. Additionally,

324 because the samples studied here are from patients with late-stage disease, it is

325 possible that tumor-induced changes to upper-gastrointestinal physiology and

326 dysphagia symptom-induced major dietary changes could themselves alter the oral

327 microbiomes of these patients. The previous findings from the ESCCAPE studies in

328 Kenya and Tanzania $(17,19)$ which found strong associations with dental staining (ORs

$329>10)$ and for which photographic validation studies suggest that most dental staining

330 was not fluorosis, also point to a recent build-up of chromogenic bacteria. Studies of the

331 oral microbiome of patients at earlier stages of ESCC and in prospective studies are

332 necessary to address this possibility. We restricted our analysis to 21 tumor-oral pairs

333 that have a sufficient number of bacterial reads (at least 10,000). It is likely that

334 excluded samples are not molecularly distinct from included samples but that the 
335 relatively low bacterial read counts in some tumors is simply reflective of low

336 sequencing depth.

338 Our observation of similar tumor and saliva microbiomes in ESCC patients is especially

339 notable considering emerging evidence linking periodontal disease and poor oral health

340 with increased risk of various cancers $(17,53,54)$. This raises several important open

341 questions. It will be essential to determine if there is a difference in the oral prevalence

342 of these identified cancer-associated bacteria between ESCC patients and non-patients

343 earlier in the natural history of the disease, for example through comparisons of patients

344 with esophageal squamous dysplasia and healthy controls. Because the prevalence of

345 these bacteria may be associated with factors such as oral health, hygiene, and diet,

346 studies of the impact of these factors on the oral microbiome in the general population

347 would inform whether the oral microbiome is on a pathway linking oral hygiene to ESCC

348 risk and may have a role in prevention.

350 In conclusion, we show that cancer-associated, traditionally-oral bacteria including the

351 genera Fusobacterium, Selenomonas, Prevotella, Streptococcus, Porphyromonas,

352 Veillonella, and Campylobacter are highly abundant within ESCC tumors from patients

353 in high-ESCC incidence regions. We also show that there is a correlation between the

354 genus composition of the saliva microbiome and the ESCC tumor microbiome of some

355 ESCC patients. These findings will be foundational for future studies to understand if

356 and how bacteria influence ESCC pathogenesis and to understand the role of the oral 
357 microbiome in this process. Finally, this study highlights the benefit of collaborative

358 investigation to evaluate the international heterogeneity of this disease.

MATERIALS AND METHODS

\section{Sample acquisition and sequencing}

363 The sample acquisition and sequencing methods for the studies from the MUHAS

364 Tanzania cohort $(n=61)(36)$ and UNC Project - Malawi cohort $(n=30)(11)$ have been

365 previously described. Samples sequenced in the Mutographs study $(n=210)(38)$

366 originated from patients in Golestan, Iran $(n=55)$, ESCCAPE case-control studies in

367 Tanzania $(n=18)(19)$ and Kenya $(n=64)(17)$, and patients in Shanxi, China $(n=71)$.

368 TCGA ESCC $(n=36)$ and COAD samples $(n=51)$ have been previously described (39,

369 41). The TCGA ESCC cohort includes tumors from patients in United States $(n=3)$,

370 Ukraine $(n=3)$, Vietnam $(n=22)$, and Russia $(n=8)$, regions which have lower incidence

371 of ESCC.

372

373 Metagenomic analysis

374 GATK-PathSeq (40) was used to conduct computational subtraction of human-mapping

375 reads from input RNAseq and WGS datasets. GATK-PathSeq works by first mapping

376 reads to a host reference database consisting of the human genome grch38 and

377 various supplemental human reference sequences. Next, non-human reads are

378 mapped against a comprehensive microbial database, and microbe read assignments

379 are reported for further study. From the MUHAS Tanzania cohort, a total of 61 tumor 
380 WGS samples, 45 saliva WGS samples, and 59 RNAseq samples were processed

381 through GATK-PathSeq.

382

383 Bacterial abundance analyses and plotting were conducted in R (v3.5.1). To calculate

384 relative abundance at a phylogenetic level (e.g., phylum or genus), GATK-PathSeq

385 results were filtered for taxa at the level, and relative abundance was calculated for

386 each taxon as follows: (\# of taxon reads)/(total \# reads at the selected phylogenetic

387 level). The rows of all bacterial abundance heatmaps are arranged according to the

388 mean abundance across all samples. The sample order of relative abundance stacked

389 barplots were determined based on Fusobacterium genus relative abundance except

390 where noted. In Figure 2D, if any cohort contained more than 50 samples, 50 samples

391 were randomly selected for plotting. The distribution of relative abundances of genera of

392 interest in all samples can be found in Figure S2, where width of each violin represents

393 the relative distribution of observed bacterial relative abundance for all patients in each

394 patient cohort.

395

396 Jaccard distance between RNAseq and WGS data from each ESCC tumor was

397 calculated in $\mathrm{R}$ based on bacterial genera with at least $1 \%$ relative abundance. The

398 qualitative Jaccard index was used in this case because the comparison was between

399 DNA and RNA analytes which would not be expected to be quantitatively identical.

400

401 Tumor-saliva similarity 
402 Only tumor-saliva pairs from the MUHAS Tanzania cohort with at least 10,000 reads

403 mapped to the bacterial superkingdom were available for analysis. This resulted in a

404 total of 21 tumor-oral pairs. Bray-Curtis dissimilarity metrics between tumor-oral pairs

405 were calculated using the R package vegan (55). Figure 3A presents the Bray-Curtis

406 similarity (1 - Bray-Curtis dissimilarity), for each tumor-oral pair.

408 To determine the correlation between the relative abundance of specific genera

409 between tumor and saliva microbiomes, common-abundant genera that are at least $1 \%$

410 abundance in at least 3 tumor-oral pairs were identified. This resulted in the

411 identification of 16 common-abundant genera. Correlations represent a two-sided

412 Pearson correlation coefficient. To determine tumor-oral enrichment of common-

413 abundant genera, the difference in relative abundance of each genus between each

414 tumor-oral pair was plotted (Figure 3C). For the relative abundance bar plots of tumor-

415 saliva pairs (Figure 3D), bacterial genera that had been highlighted in previous figures

416 are labeled.

418 Code and processed data availability

419 All GATK-PathSeq output files and reproducible analysis and plotting R Notebooks are 420 available.

421 Zenodo: https://doi.org/10.5281/zenodo.4750577

422 GitHub: https://github.com/jnoms/ESCC microbiome 
424 Furthermore, all analysis and figures can be automatically reproduced through a series

425 of Google Colab documents.

426 Figure 1 and Supplementary Figure 1:

427 https://github.com/jnoms/ESCC microbiome/blob/main/collab/Figure1.ipynb

428 Figure 2 and Supplementary Figure 2:

429 https://github.com/inoms/ESCC microbiome/blob/main/collab/Figure2.ipynb

430 Figure 3: https://github.com/inoms/ESCC microbiome/blob/main/collab/Figure3.ipynb

\section{ABBREVIATIONS}

433 AFRECC - African Esophageal Cancer Consortium

434 COAD - Colon adenocarcinoma

435 ESCA - Esophageal adenocarcinoma

436 ESCC - Esophageal squamous cell carcinoma

437 ESCCAPE - Esophageal Squamous Cell Carcinoma African Prevention Research

438 MUHAS - Muhimbili University of Health and Allied Sciences

439 RNAseq - RNA sequencing

440 TCGA - The Cancer Genome Atlas

441 WGS - Whole genome sequencing 
446 We thank Liu et al. (37) for providing access to sequencing data from ESCC patients in

447 Malawi (dbGaP accession phs001448.v1.p1). Portions of this research were conducted

448 using the $\mathrm{O} 2$ High Performance Computing Cluster, supported by the Research

449 Computing Group at Harvard Medical School. We thank Aleksandar Kostic for his

450 helpful discussions. We thank the teams from MUHAS-ORCI-UCSF Cancer

451 Collaboration, UNC-Malawi Project, Mutographs, ESCCAPE, and TCGA for specimen

452 and data collection.

\section{CONFLICTS OF INTEREST}

456 M.M. receives research support from Bayer, Novo, Ono, and Janssen, has patents

457 licensed to Bayer and Labcorp, and is a consultant for Bayer, Interline and OrigiMed.

458 J.A.D. receives research support from Constellation Pharmaceuticals and is a

459 consultant to EMD Serono, Inc. and to Merck \& Co. Inc. SB is a consultant for X-Biotix 460 and BiomX. KVL receives research funding from Celgene Cancer Carelinks ${ }^{\mathrm{TM}}$. D.L.N.

461 receives research funding from Cepheid, Inc.

\section{FUNDING SOURCES}

464 National Institutes of Health, National Cancer Institute Cancer Center Administrative 465 Supplement to Promote Cancer Prevention and Control Research in Low and Middle 466 Income Countries, A119617, [CA0082629] to K.V.L. S.B. received funding from NIH/NCI 467 Grants: R00CA229984 and Cancer Center Support Grant P30 CA015704. EAC 468 received funding from NIH/NCI Grants: U24CA210974, R01CA222862, R01CA227807, 
R01CA239604, R01CA230263. This work was supported in part by the US Public

470 Health Service grants R35CA232128 and P01CA203655 to J.A.D. Content does not

471 reflect the views of the National Cancer Institute or National Institutes of Health. This

472 work was supported by a Cancer Grand Challenges OPTIMISTICC team award

473 (C10674/A27140) to M.M. and a Cancer Grand Challenges Mutographs team award

474 funded by Cancer Research UK [C98/A24032] to M.R.S. and P.B. R21CA191965

475 supported the collection of ESCCAPE Kenya tumors. The Internatinal Agency for

476 Research on Cancer Section of Environment and Radiation supported the collection of

477 ESCCAPE Tanzania tumors. This work was supported in part by the Intramural

478 Research Program of the National Institutes of Health, National Cancer Institute,

479 Division of Cancer Epidemiology and Genetics.

FIGURE LEGENDS

Figure 1. Microbiome structure and composition of ESCC tumors

A. Description of ESCC patients, and sample types, assessed in this study. TCGA African Prevention Research; Mutographs - Cancer Research UK Mutographs Project.

B. Bacterial burden of ESCC tumors for each patient cohort. Units are bacterial reads per million human reads as determined by GATK-PathSeq analysis. Each 
dot represents one sample. Analyte type (RNA or DNA) and tumor type (ESCC or COAD) are indicated by color. was determined for each sample at the genus level based on genera that are at least $1 \%$ relative abundance. Each dot represents one sample. Analyte type (RNA or DNA) and tumor type (ESCC or COAD) are indicated by color.

D. Heatmap describing the relative abundance of the five top phyla sorted by

Figure 2. Identification of bacterial genera associated with carcinogenesis average phylum relative abundance. Each column represents one sample. Rows represent the indicated phyla. Units are relative abundance. Samples from each

A. Bacterial genera relative abundance of WGS data from the MUHAS Tanzania cohort. Each column represents a single sample. Samples are ordered by decreasing Fusobacterium relative abundance. Units are relative abundance of bacterial genus-mapping reads. Color indicates the genus, and seven genera are specified. Only patients with GATK-PathSeq analysis from both RNAseq and WGS tumor data are plotted $(n=59)$. Columns are ordered by decreasing relative abundance of Fusobacterium genus reads.

B. Bacterial genera relative abundance of RNAseq data from the MUHAS Tanzania cohort. Each column represents a single sample. Here, column order is dictated according to the patient order in Figure 2A. Units are relative abundance of bacterial genus-mapping reads. Color indicates the genus, and seven genera are 
specified. Only patients with GATK-PathSeq analysis from both RNAseq and WGS tumor data are plotted $(n=59)$. Samples are ordered in the same order as Figure $2 \mathrm{~A}$, which is by Fusobacterium genus relative abundance in the WGS data.

C. Jaccard index between RNAseq and WGS data of tumors from the MUHAS Tanzania cohort. For the "Paired by Sample" column, Jaccard indices were calculated only between the WGS and RNAseq data from the same tumor $(n=59$ comparisons). For the "Random Pairs" column, Jaccard indices were calculated between all possible WGS-RNAseq pairs independent of patient of origin to represent the expected random distribution of Jaccard indices $(n=3,481$ comparisons). Jaccard index was calculated from relative abundance at the index, and lines indicate $25^{\text {th }}, 50^{\text {th }}$, and $75^{\text {th }}$ percentiles.

528 D. Bacterial genera relative abundance of the remaining patient cohorts, including RNAseq and WGS data as indicated. Each column represents a single sample. Samples are ordered by decreasing Fusobacterium relative abundance within each patient cohort. Units are relative abundance of bacterial genus-mapping reads. Color indicates the genus, and seven genera are specified. Here, if there were more than 50 samples in a patient cohort, 50 samples were randomly selected for visualization. USA - United States, UA - Ukraine, RU - Russia. All cohorts consist of WGS data, with the exception of the tumors from Malawi which are RNAseq. (Number of samples plotted: UNC Project - Malawi 30; ESCCAPE 
Tanzania 18; ESCCAPE Kenya 50; Shanxi, China 50; Golestan, Iran 50; TCGA

540 Figure 3. Association between synchronous saliva and tumor microbiomes in

541 Tanzanian ESCC patients

542 A. Bray Curtis Similarity comparing tumor-saliva pairs from patients in the MUHAS Tanzania cohort. Analysis was restricted to the 21 tumor-saliva pairs that contained at least 10,000 bacterial reads. This analysis was conducted at the genus level and using relative abundance. For the "Paired by Patient" column, Bray Curtis Similarity was calculated only between the tumor and saliva WGS data from the same patient. For the "Random Pairs" column, Bray Curtis Similarity was calculated between all possible tumor-saliva pairs independent of patient of origin to represent the expected random distribution of Bray Curtis Similarity. ( $p=0.0003$, Wilcoxon rank sum test).

B. Correlation between the relative abundance of common-abundant bacterial genera in paired saliva and tumor WGS data. Analysis was restricted to the 21 tumor-saliva pairs that contained at least 10,000 bacterial reads. Commonabundant bacterial genera are bacterial genera that are at least $1 \%$ abundance in at least 3 tumor-saliva pairs - 16 bacterial genera made this cutoff. Correlation represents a two-sided Pearson correlation. X-axis is the correlation coefficient, and $\mathrm{Y}$ axis is the correlation $\mathrm{P}-$ Value plotted on a log scale.

C. Enrichment of genera in the oral or tumor microbiome. Each row details one of the 16 common-abundant bacterial genera. Each row contains one data point per 
patient, for a total of 21 data points. The value of each point represents the

difference in the relative abundance of the specified genus in the tumor and oral microbiomes of one patient, with positive values indicating a genus is at higher relative abundance in a patient's tumor. For example, if a genus is at a relative abundance of $0.7(70 \%)$ in the tumor and $0.3(30 \%)$ in the saliva of a patient, the plotted value for that genus and that patient is 0.4 . Curves represent the distribution of this relative abundance difference across the tumor-oral pairs, with dots indicating individual tumor-oral pairs. Vertical red lines indicate quartiles.

D. Relative abundance bar charts of tumor-saliva pairs. Analysis was restricted to the 21 tumor-saliva pairs that contained at least 10,000 bacterial reads. Units are relative abundance of bacterial genus-mapping reads. Color indicates the genus, and seven genera are specified. (abbreviations: $\mathrm{T}$ - tumor, $\mathrm{S}-$ saliva).

Figure S1. GATK-PathSeq statistics and extended phyla and genera information

A. Boxplots indicating the number of GATK-PathSeq Human-mapped reads and GATK-PathSeq microbe-mapped reads for each patient cohort. Samples from each cohort are WGS unless noted with "(RNA)", in which case they are RNAseq.

B. Heatmap describing the relative abundance of the 15 top phyla sorted by average phylum relative abundance. Each column represents one sample. Rows represent the indicated phyla. Units are relative abundance. Samples from each cohort are WGS unless noted with "(RNA)", in which case they are RNAseq. 
C. Heatmap describing the relative abundance of the 15 top genera sorted by average genera relative abundance. Each column represents one sample. Rows represent the indicated genera. Units are relative abundance. Samples from each cohort are WGS unless noted with "(RNA)", in which case they are RNAseq. Actinobacteria for each patient in each cohort. Samples from each cohort are WGS unless noted with "(RNA)", in which case they are RNAseq.

Figure S2. Distribution of Fusobacterium, Selenomonas, Prevotella,

A. The distribution of the relative abundance of genus-mapping reads for seven selected genera in all studies. The width of each violin represents the proportion of samples which have the indicated relative abundance of each genus. In contrast to Figure 2D, which only plots up to 50 samples per study, this plot includes all patients. Samples from each study are WGS unless noted with "(RNA)", in which case they are RNAseq. 


\section{REFERENCES}

604 1. Sung H, Ferlay J, Siegel RL, Laversanne M, Soerjomataram I, Jemal A, et al.

605 Global cancer statistics 2020: GLOBOCAN estimates of incidence and mortality

606 worldwide for 36 cancers in 185 countries. CA: A Cancer Journal for Clinicians.n/a(n/a).

607 2. CC MRA, Dawsey S. Oesophageal cancer: A tale of two malignancies. World

608 Cancer Report: Cancer Research for Cancer Prevention Lyon, France: International

609 Agency for Research on Cancer Available from: http://publications.iarc.fr/586. 2020.

610 3. Coleman HG, Xie S-H, Lagergren J. The epidemiology of esophageal

611 adenocarcinoma. Gastroenterology. 2018;154(2):390-405.

6124 4. Rustgi AK, El-Serag HB. Esophageal carcinoma. New England Journal of

613 Medicine. 2014;371(26):2499-509.

614 5. Arnold M, Soerjomataram I, Ferlay J, Forman D. Global incidence of

615 oesophageal cancer by histological subtype in 2012. Gut. 2015;64(3):381-7.

616 6. Cheng ML, Zhang L, Borok M, Chokunonga E, Dzamamala C, Korir A, et al. The 617 incidence of oesophageal cancer in Eastern Africa: identification of a new geographic

618 hot spot? Cancer epidemiology. 2015;39(2):143-9.

619 7. Abnet CC, Kamangar F, Islami F, Nasrollahzadeh D, Brennan P, Aghcheli K, et

620 al. Tooth loss and lack of regular oral hygiene are associated with higher risk of

621 esophageal squamous cell carcinoma. Cancer Epidemiology and Prevention

622 Biomarkers. 2008;17(11):3062-8.

623 8. Abnet CC, Qiao Y-L, Mark SD, Dong Z-W, Taylor PR, Dawsey SM. Prospective

624 study of tooth loss and incident esophageal and gastric cancers in China. Cancer

625 Causes \& Control. 2001;12(9):847-54.

626 9. Dar N, Islami F, Bhat G, Shah I, Makhdoomi M, Iqbal B, et al. Poor oral hygiene

627 and risk of esophageal squamous cell carcinoma in Kashmir. British journal of cancer.

628 2013;109(5):1367-72.

629 10. Chen X, Yuan Z, Lu M, Zhang Y, Jin L, Ye W. Poor oral health is associated with

630 an increased risk of esophageal squamous cell carcinoma-a population-based case-

631 control study in China. International journal of cancer. 2017;140(3):626-35.

632 11. Sato F, Oze I, Kawakita D, Yamamoto N, Ito H, Hosono S, et al. Inverse

633 association between toothbrushing and upper aerodigestive tract cancer risk in a

634 Japanese population. Head \& neck. 2011;33(11):1628-37.

635 12. Liang H, Yang Z, Wang JB, Yu P, Fan JH, Qiao YL, et al. Association between

636 oral leukoplakia and risk of upper gastrointestinal cancer death: a follow-up study of the

637 Linxian general population trial. Thoracic cancer. 2017;8(6):642-8.

638 13. Guha N, Boffetta P, Wünsch Filho V, Eluf Neto J, Shangina O, Zaridze D, et al.

639 Oral health and risk of squamous cell carcinoma of the head and neck and esophagus:

640 results of two multicentric case-control studies. American journal of epidemiology.

$641 \quad 2007 ; 166(10): 1159-73$.

642 14. Chen Q-L, Zeng X-T, Luo Z-X, Duan X-L, Qin J, Leng W-D. Tooth loss is

643 associated with increased risk of esophageal cancer: evidence from a meta-analysis

644 with dose-response analysis. Scientific reports. 2016;6(1):1-7.

645 15. Sheikh M, Poustchi H, Pourshams A, Etemadi A, Islami F, Khoshnia M, et al.

646 Individual and combined effects of environmental risk factors for esophageal cancer 
647 based on results from the Golestan Cohort Study. Gastroenterology. 2019;156(5):1416-

64827.

649 16. Patel K, Wakhisi J, Mining S, Mwangi A, Patel R. Esophageal cancer, the

650 topmost cancer at MTRH in the Rift Valley, Kenya, and its potential risk factors.

651 International Scholarly Research Notices. 2013;2013.

652 17. Menya D, Maina SK, Kibosia C, Kigen N, Oduor M, Some F, et al. Dental

653 fluorosis and oral health in the African Esophageal Cancer Corridor: Findings from the

654 Kenya ESCCAPE case-control study and a pan-African perspective. International

655 journal of cancer. 2019;145(1):99-109.

656 18. Mmbaga EJ, Mushi BP, Deardorff K, Mgisha W, Akoko LO, Paciorek A, et al. A

657 Case-Control Study to Evaluate Environmental and Lifestyle Risk Factors for

658 Esophageal Cancer in Tanzania. Cancer Epidemiology and Prevention Biomarkers.

6592020.

660 19. Mmbaga BT, Mwasamwaja A, Mushi G, Mremi A, Nyakunga G, Kiwelu I, et al.

661 Missing and decayed teeth, oral hygiene and dental staining in relation to esophageal

662 cancer risk: ESCCAPE case-control study in Kilimanjaro, Tanzania. International journal

663 of cancer. 2020.

664 20. Buckle GC, et al. Risk factors associated with early-onset esophageal cancer in

665 Tanzania. (Under Review).

666 21. Ishaq S, Nunn L. Helicobacter pylori and gastric cancer: a state of the art review.

667 Gastroenterology and hepatology from bed to bench. 2015;8(Suppl1):S6.

668 22. Kostic AD, Chun E, Robertson L, Glickman JN, Gallini CA, Michaud M, et al.

669 Fusobacterium nucleatum potentiates intestinal tumorigenesis and modulates the

670 tumor-immune microenvironment. Cell host \& microbe. 2013;14(2):207-15.

671 23. Kostic AD, Gevers D, Pedamallu CS, Michaud M, Duke F, Earl AM, et al.

672 Genomic analysis identifies association of Fusobacterium with colorectal carcinoma.

673 Genome research. 2012;22(2):292-8.

674 24. Castellarin M, Warren RL, Freeman JD, Dreolini L, Krzywinski M, Strauss J, et al.

675 Fusobacterium nucleatum infection is prevalent in human colorectal carcinoma.

676 Genome research. 2012;22(2):299-306.

677 25. Bullman S, Pedamallu CS, Sicinska E, Clancy TE, Zhang X, Cai D, et al.

678 Analysis of Fusobacterium persistence and antibiotic response in colorectal cancer.

679 Science. 2017;358(6369):1443-8.

680 26. Gur C, Ibrahim Y, Isaacson B, Yamin R, Abed J, Gamliel M, et al. Binding of the

681 Fap2 protein of Fusobacterium nucleatum to human inhibitory receptor TIGIT protects

682 tumors from immune cell attack. Immunity. 2015;42(2):344-55.

683 27. Rubinstein MR, Baik JE, Lagana SM, Han RP, Raab WJ, Sahoo D, et al.

684 Fusobacterium nucleatum promotes colorectal cancer by inducing Wnt/ $\beta$-catenin

685 modulator Annexin A1. EMBO reports. 2019;20(4):e47638.

686 28. Rubinstein MR, Wang X, Liu W, Hao Y, Cai G, Han YW. Fusobacterium

687 nucleatum promotes colorectal carcinogenesis by modulating E-cadherin/ $\beta$-catenin

688 signaling via its FadA adhesin. Cell host \& microbe. 2013;14(2):195-206.

689 29. Gao S, Li S, Ma Z, Liang S, Shan T, Zhang M, et al. Presence of Porphyromonas

690 gingivalis in esophagus and its association with the clinicopathological characteristics

691 and survival in patients with esophageal cancer. Infectious agents and cancer.

692 2016;11(1):3. 
693 30. Whitmore SE, Lamont RJ. Oral bacteria and cancer. PLoS pathogens.

694 2014;10(3):e1003933.

695 31. Inaba H, Sugita H, Kuboniwa M, Iwai S, Hamada M, Noda T, et al. P

696 orphyromonas gingivalis promotes invasion of oral squamous cell carcinoma through

697 induction of pro MMP 9 and its activation. Cellular microbiology. 2014;16(1):131-45.

698 32. He Z, Gharaibeh RZ, Newsome RC, Pope JL, Dougherty MW, Tomkovich S, et

699 al. Campylobacter jejuni promotes colorectal tumorigenesis through the action of

700 cytolethal distending toxin. Gut. 2019;68(2):289-300.

701 33. Narikiyo M, Tanabe C, Yamada Y, Igaki H, Tachimori Y, Kato H, et al. Frequent

702

703

704

705

706

707

708 and preferential infection of Treponema denticola, Streptococcus mitis, and Streptococcus anginosus in esophageal cancers. Cancer science. 2004;95(7):569-74. 34. Boleij A, Schaeps RM, Tjalsma H. Association between Streptococcus bovis and colon cancer. Journal of clinical microbiology. 2009;47(2):516-.

35. Ferrari A, Botrugno I, Bombelli E, Dominioni T, Cavazzi E, Dionigi P. Colonoscopy is mandatory after Streptococcus bovis endocarditis: a lesson still not learned. Case report. World journal of surgical oncology. 2008;6(1):49. 36. Van Loon K, et al. A Genomic Analysis of Esophageal Squamous Cell Carcinoma in Eastern Africa. (Under Review).

37. Liu W, Snell JM, Jeck WR, Hoadley KA, Wilkerson MD, Parker JS, et al. Subtyping sub-Saharan esophageal squamous cell carcinoma by comprehensive molecular analysis. JCI insight. 2016;1(16).

38. Moody S, Senkin S, Islam SMA, Wang J, Nasrollahzadeh D, Penha RCC, et al. Mutational signatures in esophageal squamous cell carcinoma from eight countries of varying incidence. medRxiv. 2021:2021.04.29.21255920.

39. Network CGAR. Integrated genomic characterization of oesophageal carcinoma. Nature. 2017;541(7636):169.

40. Walker MA, Pedamallu CS, Ojesina AI, Bullman S, Sharpe T, Whelan CW, et al. GATK PathSeq: a customizable computational tool for the discovery and identification of microbial sequences in libraries from eukaryotic hosts. Bioinformatics.

2018;34(24):4287-9.

41. Network CGA. Comprehensive molecular characterization of human colon and rectal cancer. Nature. 2012;487(7407):330.

42. Dohlman AB, Arguijo Mendoza D, Ding S, Gao M, Dressman H, Iliev ID, et al. The cancer microbiome atlas: a pan-cancer comparative analysis to distinguish tissueresident microbiota from contaminants. Cell Host \& Microbe. 2020.

43. Tsay J-CJ, Wu BG, Sulaiman I, Gershner K, Schluger R, Li Y, et al. Lower airway dysbiosis affects lung cancer progression. Cancer Discovery. 2020.

730 44. Ricotta C, Podani J. On some properties of the Bray-Curtis dissimilarity and their 731 ecological meaning. Ecological Complexity. 2017;31:201-5.

732 45. Flemer B, Warren RD, Barrett MP, Cisek K, Das A, Jeffery IB, et al. The oral

733 microbiota in colorectal cancer is distinctive and predictive. Gut. 2018;67(8):1454-63.

734 46. Komiya Y, Shimomura Y, Higurashi T, Sugi Y, Arimoto J, Umezawa S, et al.

735 Patients with colorectal cancer have identical strains of Fusobacterium nucleatum in

736 their colorectal cancer and oral cavity. Gut. 2019;68(7):1335-7. 
737 47. Yamamura K, Baba Y, Nakagawa S, Mima K, Miyake K, Nakamura K, et al.

738 Human microbiome Fusobacterium nucleatum in esophageal cancer tissue is

739 associated with prognosis. Clinical Cancer Research. 2016;22(22):5574-81.

740 48. Peters BA, Wu J, Pei Z, Yang L, Purdue MP, Freedman ND, et al. Oral

741 microbiome composition reflects prospective risk for esophageal cancers. Cancer

742 research. 2017;77(23):6777-87.

743 49. Kawasaki M, Ikeda Y, Ikeda E, Takahashi M, Tanaka D, Nakajima Y, et al. Oral

744 infectious bacteria in dental plaque and saliva as risk factors in patients with esophageal

745 cancer. Cancer. 2021;127(4):512-9.

746 50. Corning B, Copland AP, Frye JW. The esophageal microbiome in health and

747 disease. Current gastroenterology reports. 2018;20(8):1-7.

748 51. Dewhirst FE, Chen T, Izard J, Paster BJ, Tanner AC, Yu W-H, et al. The human

749 oral microbiome. Journal of bacteriology. 2010;192(19):5002-17.

750 52. Signat B, Roques C, Poulet P, Duffaut D. Role of Fusobacterium nucleatum in

751 periodontal health and disease. Curr Issues Mol Biol. 2011;13(2):25-36.

752 53. Michaud DS, Lu J, Peacock-Villada AY, Barber JR, Joshu CE, Prizment AE, et

753 al. Periodontal disease assessed using clinical dental measurements and cancer risk in

754 the ARIC study. JNCI: Journal of the National Cancer Institute. 2018;110(8):843-54.

755 54. Ahrens W, Pohlabeln H, Foraita R, Nelis M, Lagiou P, Lagiou A, et al. Oral

756 health, dental care and mouthwash associated with upper aerodigestive tract cancer

757 risk in Europe: the ARCAGE study. Oral oncology. 2014;50(6):616-25.

758 55. Oksanen J, Kindt R, Legendre P, O'Hara B, Stevens MHH, Oksanen MJ, et al.

759 The vegan package. Community ecology package. 2007;10:631-7. 
bioRxiv preprint doi: https://doi.org/10.1101/2021.09.29.462325; this version posted September 29, 2021. The copyright holder for this preprint Figure 1 (which was not certified by peer review) is the author/funder, who has granted bioRxiv a license to display the preprint in perpetuity. It is made

A available under acC-BY 4.0 International lice

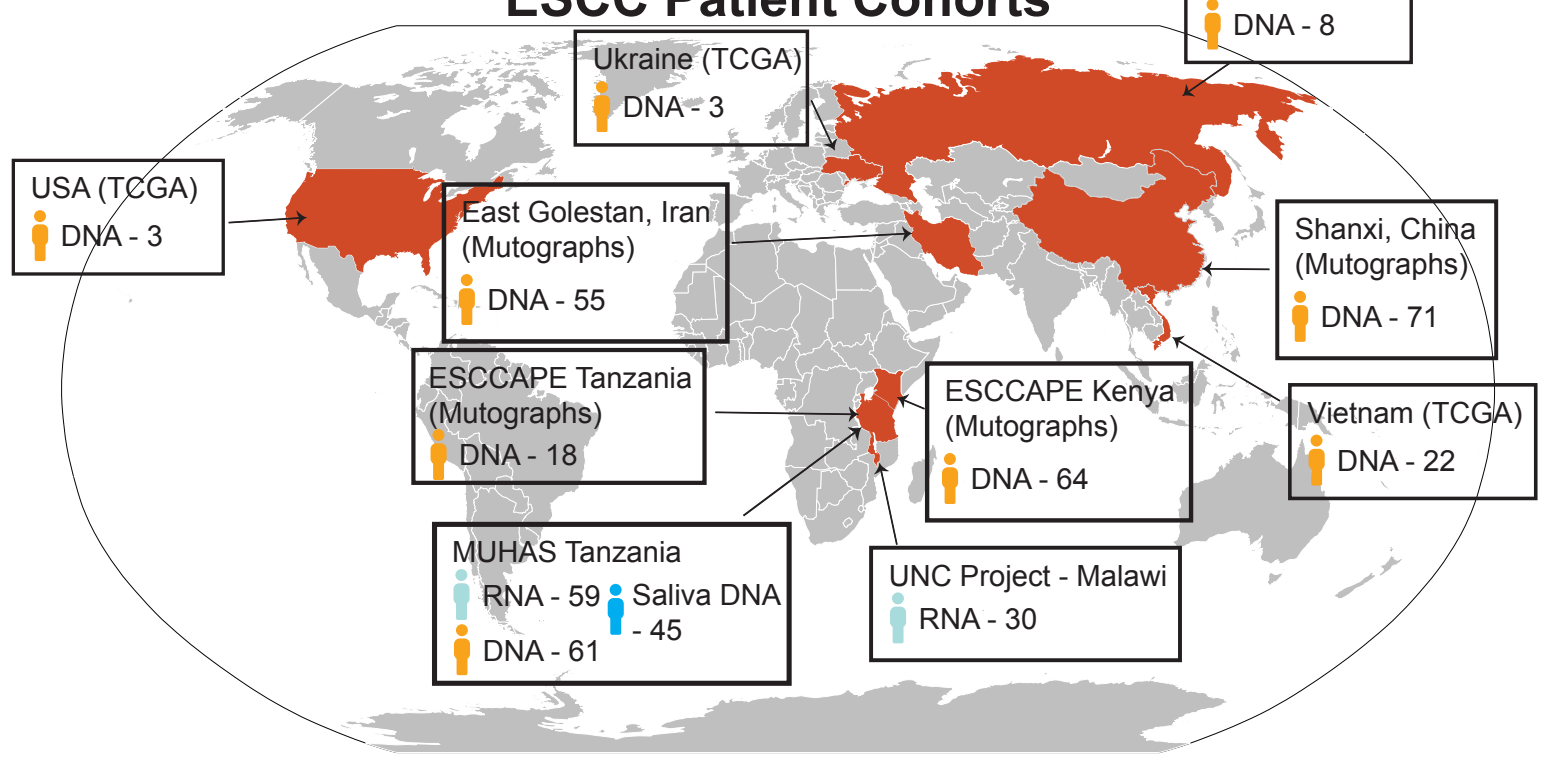

B

$$
\text { UNC }
$$

UNC Project - Malawi (RNA)

MUHAS Tanzania (RNA), MUHAS Tanzania :

ESCCAPE Tanzania

ESCCAPE Kenya

Shanxi, China

Golestan, Iran

TCGA ESCC

TCGA COAD

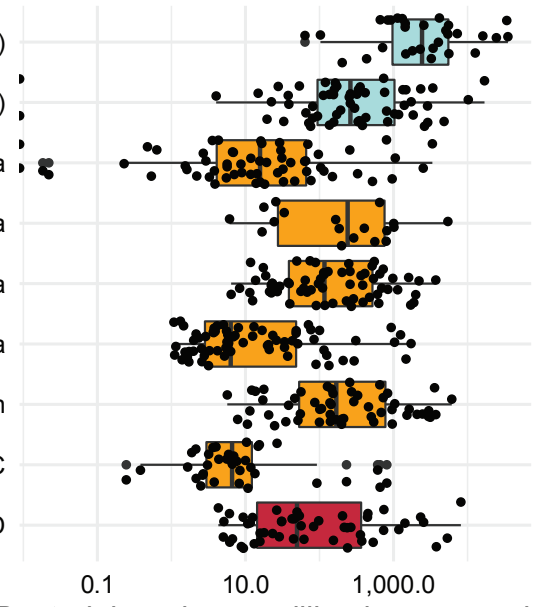

Bacterial reads per million human reads.

C

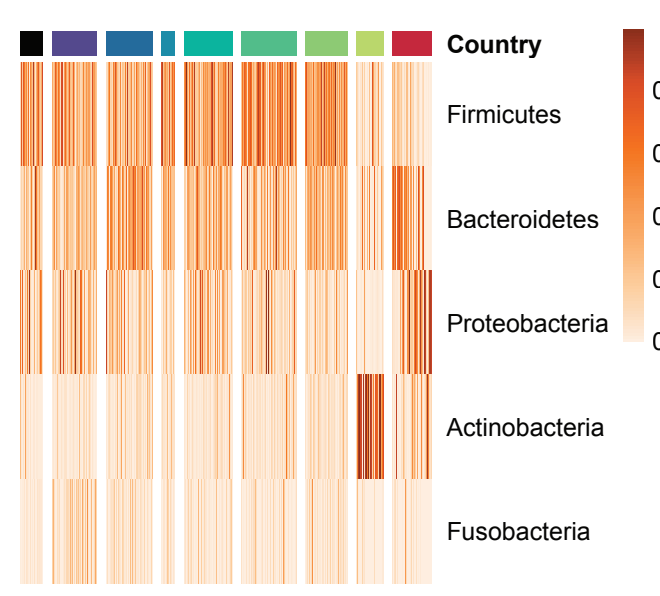

Country

Firmicutes

Bacteroidetes
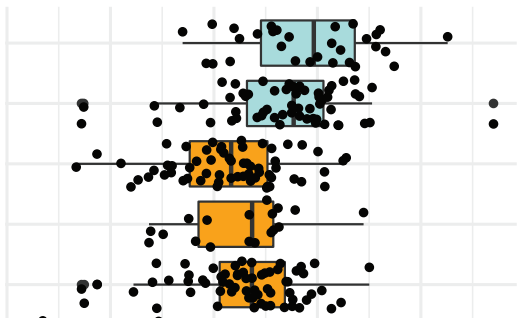

Sample_type

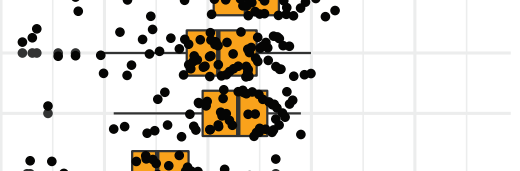

早 ESCC RNA

白 ESCC DNA

COAD DNA

D

Figure 1. Microbiome structure and composition of ESCC tumors.

A. Description of ESCC patients, and sample types, assessed in this study. TCGA - The Cancer Genome Atlas; ESCCAPE - Esophageal Squamous Cell Carcinoma African Prevention Research; Mutographs - Cancer Research UK Mutographs Project.

B. Bacterial burden of ESCC tumors for each patient cohort. Units are bacterial reads per million human reads as determined by GATK-PathSeq analysis. Each dot represents one sample. Analyte type (RNA or DNA) and tumor type (ESCC or COAD) are indicated by color.

C. Shannon diversity of ESCC tumors for each patient cohort. Shannon diversity was determined for each sample at the genus level based on genera that are at least $1 \%$ relative abundance. Each dot represents one sample. Analyte type (RNA or DNA) and tumor type (ESCC or (OAD) are indicated by color.

D. Heatmap describing the relative abundance of the five top phyla sorted by average phylum relative abundance. Each column represents one sample. Rows represent the indicated phyla. Units are relative abundance. Samples from each cohort are WGS unless noted with "(RNA)", in which case they are RNAseq. 
bioRxiv preprint doi: https://doi.org/10.1101/2021.09.29.462325; this version posted September 29, 2021. The copyright holder for this preprint Figure 2 (which was not certified by peer review) is the author/funder, who has granted bioRxiv a license to display the preprint in perpetuity. It is made

A

\section{MUHAS Tanzania (DNA)}

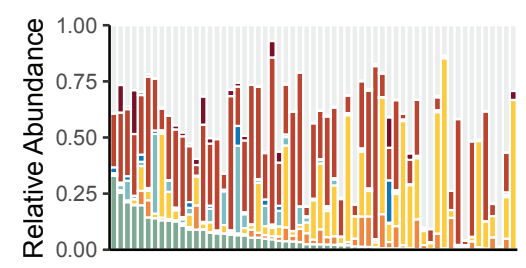

B

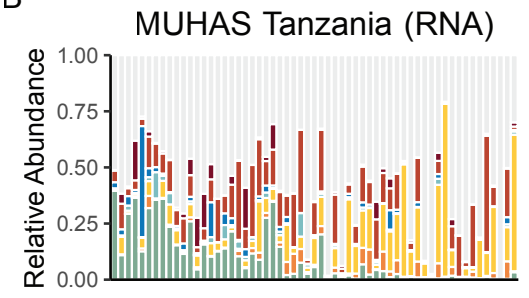

C

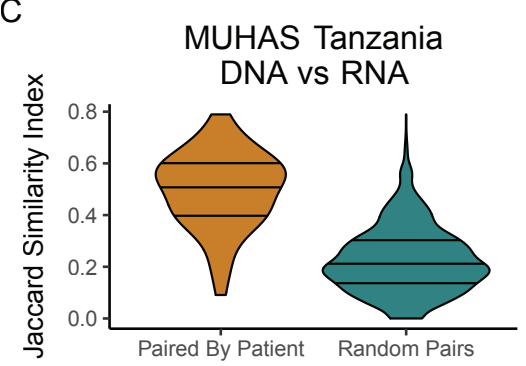

D
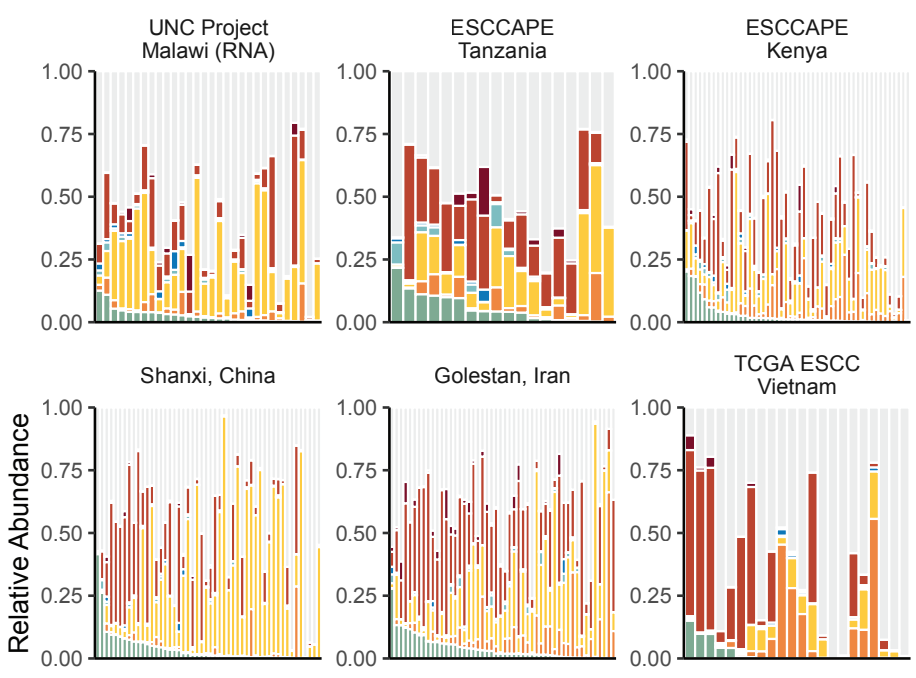

Golestan, Iran
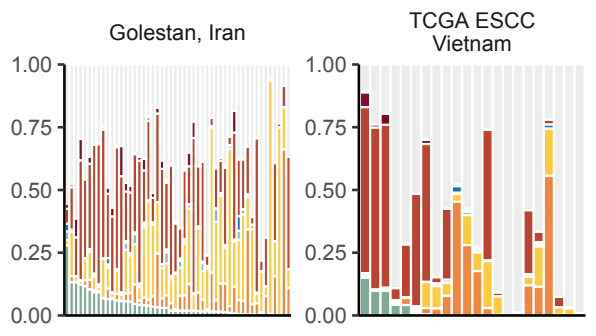

Genus

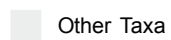

Selenomonas

Prevotella

Campylobacter

Porphyromonas

Streptococcus

Veillonella

Fusobacterium

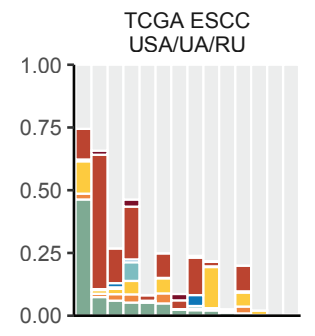

TCGA COAD

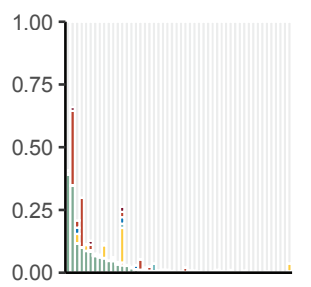

Figure 2. Identification of bacterial genera associated with carcinogenesis.

A. Bacterial genera relative abundance of WGS data from the MUHAS Tanzania cohort. Each column represents a single sample. Samples are ordered by decreasing Fusobacterium relative abundance. Units are relative abundance of bacterial genus-mapping reads. Color indicates the genus, and seven genera are specified. Only patients with GATK-PathSeq analysis from both RNAseq and WGS tumor data are plotted ( $\mathrm{n}=59$ ). Columns are ordered by decreasing relative abundance of Fusobacterium genus reads.

B. Bacterial genera relative abundance of RNAseq data from the MUHAS Tanzania cohort. Each column represents a single sample. Here, column order is dictated according to the patient order in Figure 2A. Units are relative abundance of bacterial genus-mapping reads. Color indicates the genus, and seven genera are specified. Only patients with GATK-PathSeq analysis from both RNAseq and WGS tumor data are plotted ( $\mathrm{n}=59$ ). Samples are ordered in the same order as Figure 2A, which is by Fusobacterium genus relative abundance in the WGS data.

C. Jaccard index between RNAseq and WGS data of tumors from the MUHAS Tanzania cohort. For the "Paired by Sample" column, Jaccard indices were calculated only between the WGS and RNAseq data from the same tumor ( $n=59$ comparisons). For the "Random Pairs" column, Jaccard indices were calculated between all possible WGS-RNAseq pairs independent of patient of origin to represent the expected random distribution of Jaccard indices ( $n=3,481$ comparisons). Jaccard index was calculated from relative abundance at the genus level based on genera that are at least $1 \%$ relative abundance. The width of the violin represents the relative proportion of comparisons with each Jaccard index, and lines indicate 25th, 50th, and 75th percentiles.

D. Bacterial genera relative abundance of the remaining patient cohorts, including RNAseq and WGS data as indicated. Each column represents a single sample. Samples are ordered by decreasing Fusobacterium relative abundance within each patient cohort. Units are relative abundance of bacterial genus-mapping reads. Color indicates the genus, and seven genera are specified. Here, if there were more than 50 samples in a patient cohort, 50 samples were randomly selected for visualization. USA - United States, UA - Ukraine, RU - Russia. All cohorts consist of WGS data, with the exception of the tumors from Malawi which are RNAseq. (Number of samples plotted: UNC Project - Malawi 30; ESCCAPE Tanzania 18; ESCCAPE Kenya 50; Shanxi, China 50; Golestan, Iran 50; TCGA ESCC Vietnam 22; TCGA ESCC USA/UA/RU 14). 
bioRxiv preprint doi: https://doi.org/10.1101/2021.09.29.462325; this version posted September 29, 2021. The copyright holder for this preprint (which was not certified by peer review) is the author/funder, who has granted bioRxiv a license to display the preprint in perpetuity. It is made Figure 3

A

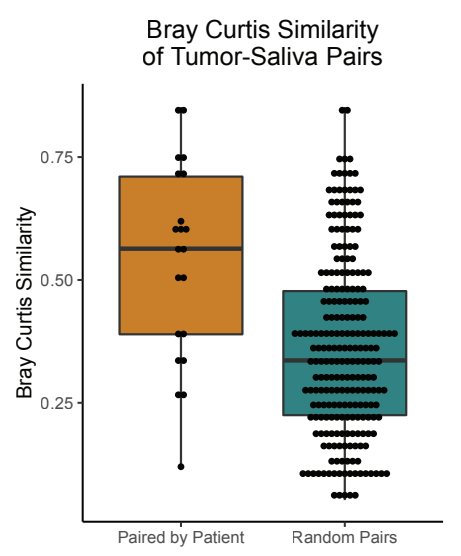

B

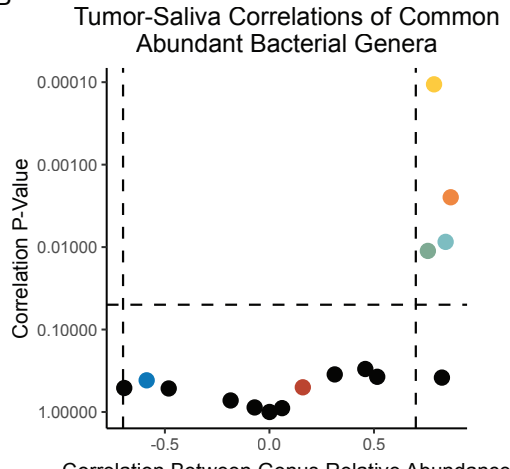

Correlation Between Genus Relative Abundance In Tumor And Saliva
C

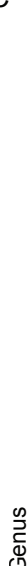

D

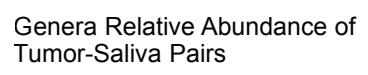

Genera Relative Abundance of Tumor-Saliva Pairs
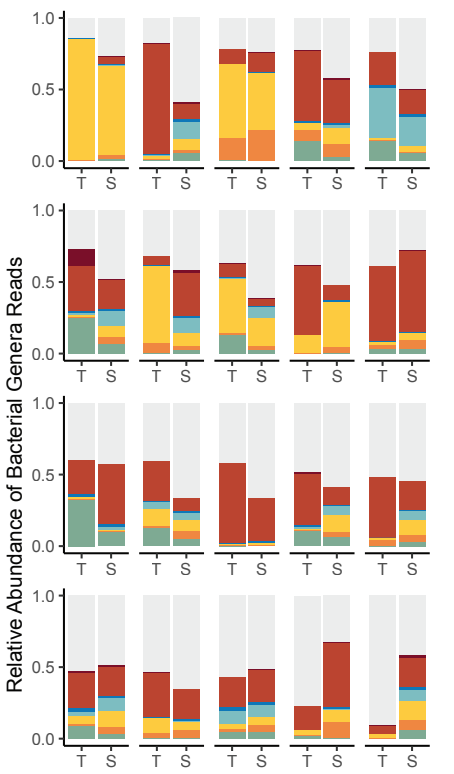

Genus
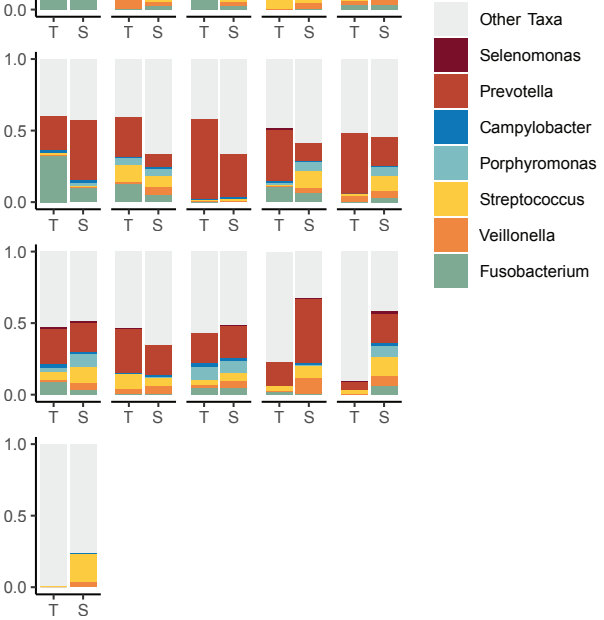

Figure 3. Association between synchronous saliva and tumor microbiomes in Tanzanian ESCC patients.

A. Bray Curtis Similarity comparing tumor-saliva pairs from patients in the MUHAS Tanzania cohort. Analysis was restricted to the 21 tumor-saliva pairs that contained at least 10,000 bacterial reads. This analysis was conducted at the genus level and using relative abundance. For the "Paired by Patient" column, Bray Curtis Similarity was calculated only between the tumor and saliva WGS data from the same patient. For the "Random Pairs" column, Bray Curtis Similarity was calculated between all possible tumor-saliva pairs independent of patient of origin to represent the expected random distribution of Bray Curtis Similarity. ( $p=0.0003$, Wilcoxon rank sum test).

B. Correlation between the relative abundance of common-abundant bacterial genera in paired saliva and tumor WGS data. Analysis was restricted to the 21 tumor-saliva pairs that contained at least 10,000 bacterial reads. Common-abundant bacterial genera are bacterial genera that are at least $1 \%$ abundance in at least 3 tumor-saliva pairs - 16 bacterial genera made this cutoff. Correlation represents a two-sided Pearson correlation. $\mathrm{X}$-axis is the correlation coefficient, and $\mathrm{Y}$ axis is the correlation $\mathrm{P}$-Value plotted on a log scale.

C. Enrichment of genera in the oral or tumor microbiome. Each row details one of the 16 common-abundant bacterial genera. Each row contains one data point per patient, for a total of 21 data points. The value of each point represents the difference in the relative abundance of the specified genus in the tumor and oral microbiomes of one patient, with positive values indicating a genus is at higher relative abundance in a patient's tumor. For example, if a genus is at a relative abundance of $0.7(70 \%)$ in the tumor and $0.3(30 \%)$ in the saliva of a patient, the plotted value for that genus and that patient is 0.4 . Curves represent the distribution of this relative abundance difference across the tumor-oral pairs, with dots indicating individual tumor-oral pairs. Vertical red lines indicate quartiles.

D. Relative abundance barcharts of tumor-saliva pairs. Analysis was restricted to the 21 tumor-saliva pairs that contained at least 10,000 bacterial eads. Units are relative abundance of bacterial genus-mapping reads. Color indicates the genus, and seven genera are specified. (Abbreviations: $\mathrm{T}$ - tumor, S- saliva.) 
bioRxiv preprint doi: https://doi.org/10.1101/2021.09.29.462325; this version posted September 29, 2021. The copyright holder for this preprint Figure (jwfich was not certified by peer review) is the author/funder, who has granted bioRxiv a license to display the preprint in perpetuity. It is made

A
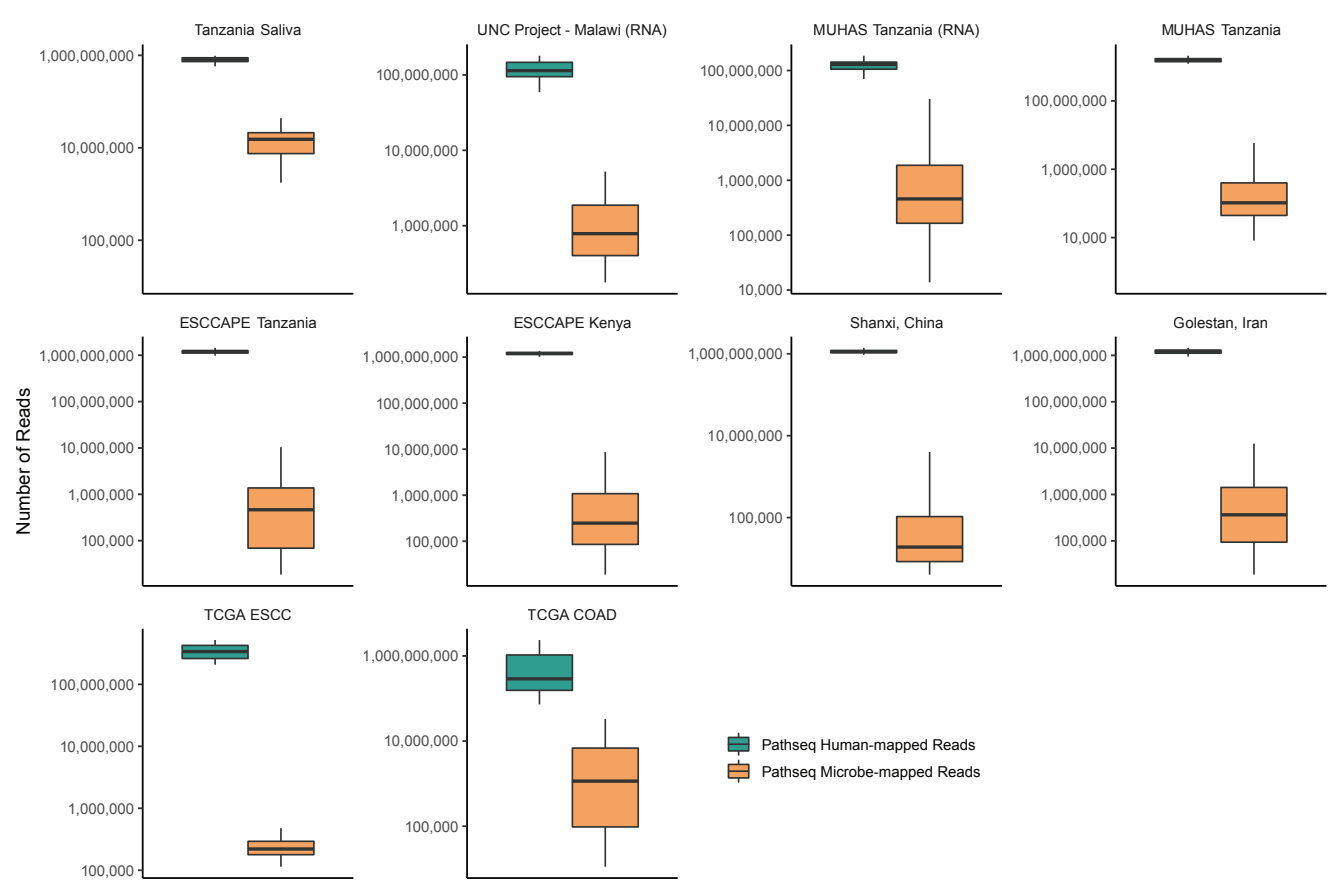

Pathseq Human-mapped Reads

B

Top 15 Phyla

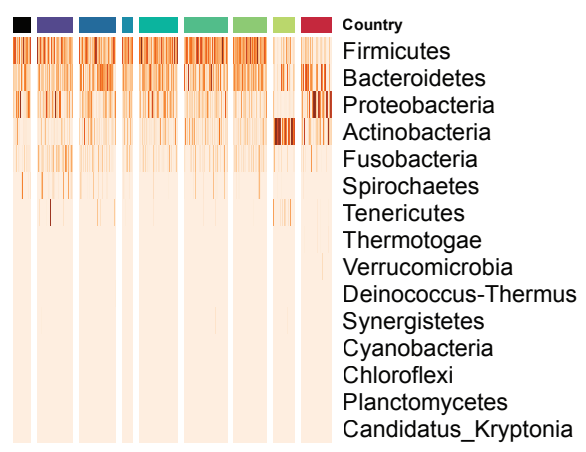

C Top 15 Genera

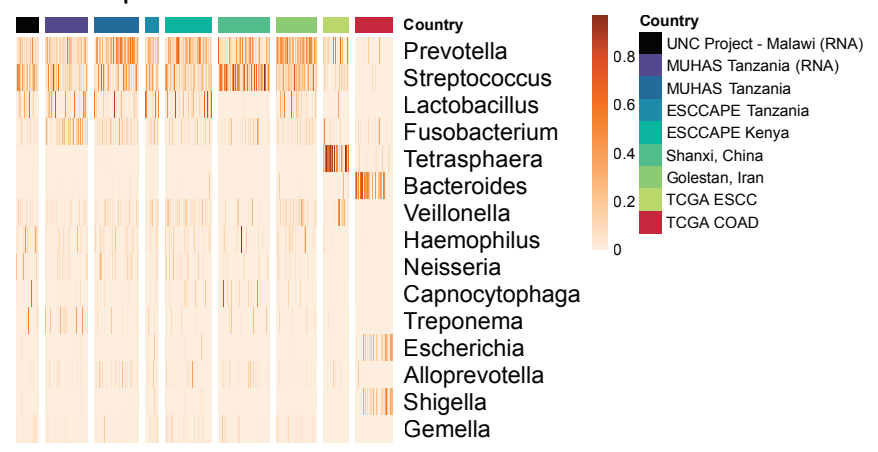

$\mathrm{D}$

Shannon Diversity Within

The Phylum Actinobacteria

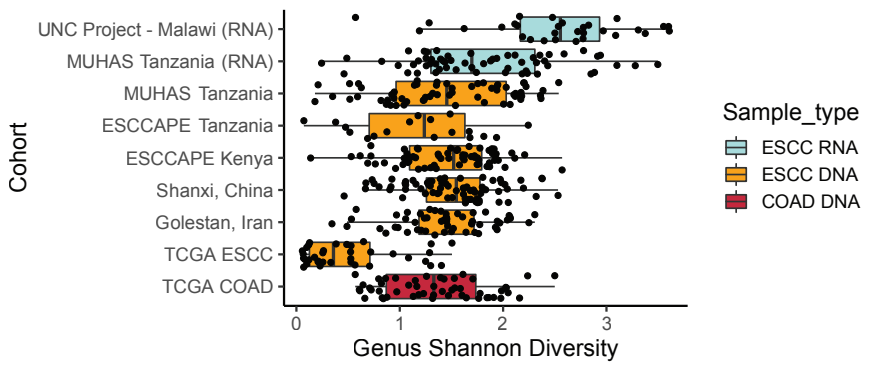

Figure S1. GATK-PathSeq statistics and extended phyla and genera information.

A. $\quad$ Boxplots indicating the number of GATK-PathSeq Human-mapped reads and GATK-PathSeq microbe-mapped reads for each patient cohort. Samples from each cohort are WGS unless noted with "(RNA)", in which case they are RNAseq.

B. Heatmap describing the relative abundance of the 15 top phyla sorted by average phylum relative abundance. Each column represents one sample. Rows represent the indicated phyla. Units are relative abundance. Samples from each cohort are WGS unless noted with "(RNA)", in which case they are RNAseq.

C. Heatmap describing the relative abundance of the 15 top genera sorted by average genera relative abundance. Each column represents one sample. Rows represent the indicated genera. Units are relative abundance. Samples from each cohort are WGS unless noted with "(RNA)", in which case they are RNAseq.

D. Boxplot representing the Shannon diversity of genera that fall within the phylum Actinobacteria for each patient in each cohort. Samples from each cohort are WGS unless noted with "(RNA)", in which case they are RNAseq. 
bioRxiy preprint doi: https://doi.org/10.1101/2021.09.29.462325; this version posted September 29, 2021. The copyright holder for this preprint Figu(horielf was not certified by peer review) is the author/funder, who has granted bioRxiv a license to display the preprint in perpetuity. It is made

A available under aCC-BY 4.0 International license.

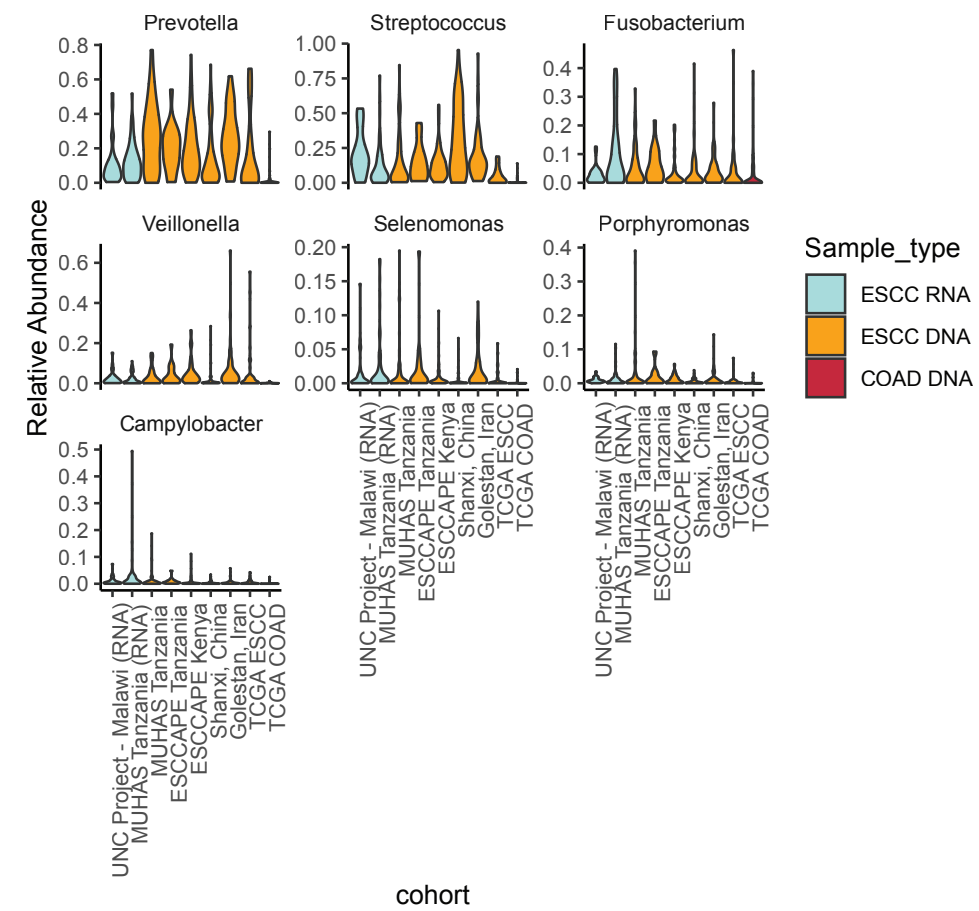

Figure S2. Distribution of Fusobacterium, Selenomonas, Prevotella, Streptococcus, Porphyromonas, Veillonella, and Campylobacter relative abundance of genus reads for all samples in each study.

A. The distribution of the relative abundance of genus-mapping reads for seven selected genera in all studies. The width of each violin represents the proportion of samples which have the indicated relative abundance of each genus. In contrast to Figure 2D, which only plots up to 50 samples per study, this plot includes all patients. Samples from each study are WGS unless noted with "(RNA)", in which case they are RNAseq. 


\begin{tabular}{|c|c|c|c|c|c|c|}
\hline \multicolumn{7}{|l|}{ TABLE 1} \\
\hline Study & Tanzania & Malawi** & $\begin{array}{c}\text { ESCCAPE } \\
\text { Tanzania*** }\end{array}$ & $\begin{array}{l}\text { ESCCAPE } \\
\text { Kenya*** }\end{array}$ & $\begin{array}{l}\text { East } \\
\text { Golestan, } \\
\text { Iran**** }\end{array}$ & $\begin{array}{c}\text { Shanxi, } \\
\text { China**** }\end{array}$ \\
\hline No. cases included & 61 & 30 & 18 & 65 & 55 & 71 \\
\hline \multicolumn{7}{|l|}{ Demographics } \\
\hline Median age (IQR) & $49(44-62)$ & 56 & $65(61-73)$ & $64(53,71)$ & $62(54,73)$ & $56(50,64)$ \\
\hline$\%$ male & $67 \%$ & $45.8 \%$ & $61 \%$ & $68 \%$ & $55 \%$ & $56 \%$ \\
\hline \multicolumn{7}{|l|}{ Status at diagnosis } \\
\hline Weight (kg), median (IQR) & & & $44(40-52)$ & $52(46,60)$ & & \\
\hline Body mass index $\left(\mathrm{kg} / \mathrm{m}^{2}\right)$ median (IQR) & & & $15.8(15.4,19.1)$ & $19.5(15.6,22.0)$ & & \\
\hline $\begin{array}{l}\text { Median months ill before coming to } \\
\text { endoscopy (IQR) }\end{array}$ & & & $2(1,6)$ & $3(2,4.5)$ & & \\
\hline \begin{tabular}{|r|r|} 
HIV status: & Positive \\
& Negative \\
& Not known
\end{tabular} & $\begin{array}{c}2(3.2 \%) \\
36(59.0 \%) \\
23(37.7 \%)\end{array}$ & $\begin{array}{c}10(16.9 \%) \\
44(74.6 \%) \\
5(8.5 \%)\end{array}$ & $\begin{array}{c}1(5 \%) \\
10(56 \%) \\
7(39 \%)\end{array}$ & $\begin{array}{c}5(8 \%) \\
48(74 \%) \\
12(18 \%)\end{array}$ & & \\
\hline \multicolumn{7}{|l|}{ Key lifestyle habits } \\
\hline $\mathrm{N}(\%)$ ever tobacco users & & & $11(61 \%)$ & $38(58 \%)$ & $17(31 \%)$ & 35 (49\%) \\
\hline $\begin{array}{r}\mathrm{N}(\%) \text { who brush teeth daily: } \\
\text { With toothbrush } \\
\text { With stick }\end{array}$ & & & $\begin{array}{l}12(67 \%) \\
6(33 \%)\end{array}$ & $\begin{array}{c}16(25 \%)^{*} \\
10(15 \%)\end{array}$ & & \\
\hline Median no missing teeth (IQR) & & & $3(1,5)$ & $4(1,8)$ & & \\
\hline
\end{tabular}

${ }^{*} \mathrm{~N}=22$ (34\%) brush once per week or never, $\mathrm{n}=17$ (26\%) brush 2 to 6 times/week

**Indicates demographics are from the entire patient population, consisting of both included and unincluded patients.

***Indicates demographic percentages are from the entire patient population, with discrete counts scaled to the number of cases included.

$* * * *$ Indicates demographic information is exclusively for included patients. 